\title{
DESARROLLANDO AUTONOMÍA EN LOS NIÑOS A TRAVÉS DEL JUEGO DIDÁCTICO
}

DEVELOPING AUTONOMY IN CHILDREN THROUGH EDUCATIONAL GAME

\section{Cruz Antonio Lip Licham, Ph.D.}

https://orcid.org/0000-0002-9670-8980

Universidad César Vallejo, Lima, Perú.

cantlip@gmail.com

\section{ARTÍCULO DE REFLEXIÓN}

Recibido: 24 de agosto de 2021

Aceptado: 28 de octubre de 2021

\section{RESUMEN}

El objetivo del presente estudio fue suscitar la reflexión del autor mediante la revisión, el análisis e interpretación de la literatura especializada en sus temas centrales: el juego, el juego didáctico y la autonomía en los niños; reflexión que se plasmó en toda la redacción del artículo y pretende hacer un llamado a padres, maestros y autoridades, para priorizar el desarrollo del nivel de autonomía de los niños y a su vez, servir como una fuente sistematizada de información relevante en estos temas. Metodológicamente tiene un enfoque cualitativo e interpretativo con un modelo de revisión literaria crítica y reflexiva basándose en la búsqueda de información en bases de datos especializadas como Scopus, Ebsco, Dialnet, etc. y el apoyo del buscador de Google Académico. Se concluyó que la autonomía debe formarse desde edades tempranas, siendo el juego el mejor medio para acelerar el desarrollo del nivel de autonomía y que el juego didáctico, es una importante estrategia pedagógica que favorece el desarrollo cognitivo, psicomotor y social de los niños al permitirle aprendizajes significativos.

Palabras claves: juego, juego didáctico, autonomía, aprendizaje significativo.

\section{ABSTRACT}

The objective of the present study was to provoke the author's reflection through the review, analysis and interpretation of the specialized literature on its central themes: play, didactic play and autonomy in children; reflection that was reflected throughout the writing of the article and aims to call on parents, teachers and authorities to prioritize the development of the level of autonomy of children and, in turn, serve as a systematized source of relevant information on these issues. Methodologically it has a qualitative and interpretative approach with a critical and reflective literature review model based on the 
search for information in specialized databases such as Scopus, Ebsco, Dialnet, etc. and the support of the Google Scholar search engine. It was concluded that autonomy should be formed from early ages, being play the best means to accelerate the development of the level of autonomy and that didactic play is an important pedagogical strategy that favors the cognitive, psychomotor and social development of children by allowing significant learning.

Keywords: game, didactic game, autonomy, significant learning.

\section{INTRODUCCIÓN}

Al aceptar que la autonomía es el derecho y la capacidad más importante en el desarrollo del ser humano, en cualquier contexto sociocultural, porque le permite autenticidad, crecimiento y libertad, en un clima de respeto a las personas y normas de convivencia; es menester hacer una reflexión crítica y reflexiva, sobre la importancia de desarrollar la autonomía desde los primeros años de vida y del cómo el juego se debe emplear como herramienta didáctica en el desarrollo de esta en los infantes; por lo que este estudio no sólo pretende servir como fuente de información actualizada del cómo y por qué se debe potenciar el desarrollo de la autonomía desde el nivel preescolar, sino que también invita, a todos los actores educativos involucrados, al accionar directo y competente en pro del desarrollo de los niños y por consiguiente de la sociedad en general; por tanto la relevancia de este estudio se refleja en la incesante búsqueda de una educación productiva y de calidad para los pequeños estudiantes en todo lugar y nivel y, se ampara en la política que expresa el convencimiento de que en la primera infancia, el autodescubrimiento, el desarrollo corporal, de la consciencia y el control psicomotor, mejoran la autonomía y seguridad personal de los niños, por tanto favorecen el desarrollo de su empatía y capacidad para las relaciones sociales y es por eso que privilegia el juego, la exploración, el descubrimiento como dinamizadores del aprendizaje (MINEDU, 2017)

Esto es consecuente con Ochoa (2017) para quien la autonomía es un elemento importante y necesario en la formación multifacética y el desarrollo integral de la personalidad de todo ser humano, porque le faculta el autogobernarse, decidir y asumir responsablemente las consecuencias de su accionar dentro de la sociedad; por tanto es un indispensable se inicie y refuerce su desarrollo desde la primera infancia, de forma gradual y sistemática, hasta conseguir personas valorativas que puedan contribuir a lograr una sociedad con justicia, paz y hermandad; más allá de fronteras, credos y razas; y con Sarramona (2012) asevera que la autonomía está completamente integrada a las tendencias educativas actuales en todos los países, pues conllevan a garantizar calidad, 
equidad y aceptación a una mayor diversidad; la misma que puede dar respuestas coherentes y específicas a requerimientos de cada caso o lugar y, por su parte, Maso (2011) la enfoca en una perspectiva mucho más amplia y recalca que la autonomía es un principio moral que ha llegado a ser una base relevante de todos los sistemas éticos y filosóficos del mundo actual.

\section{REVISIÓN TEÓRICA}

\section{El juego y su importancia formativa}

Aunque no tiene una definición totalmente aceptada, pero en base a los múltiples conceptos vertidos en Ruiz (2017) y en Secadas (1978) es posible concluir que el juego en los niños, es una actividad psicomotora compleja, innata y divertida, que se desarrolla en un contexto espacial y temporal determinado, pero sin compromiso formal con la realidad, a la que puede desfigurar a voluntad y que su única finalidad es el juego en sí mismo; por tanto tiene normas específicas para cada caso, las que son libremente elaboradas y aceptadas por los participantes. Consideran los autores que es el medio ideal de desarrollo humano en edades tempranas.

Para Cuellar et al (2018) la manera como el niño se relaciona y percibe las reglas del juego puede señalar el modo cómo el concepto de norma social evoluciona en su desarrollo personal; en este desarrollo se manifiesta la importancia de aspectos emocionales, por lo que la configuración de ambientes saludables y armónicos promueve relaciones adecuadas, que permiten obtener autoconocimiento, crecimiento y un desarrollo adecuado de la estructura física y emocional.

En cuanto a sus requisitos o características, se pueden destacar a Huizinga (2014), quien considera que este tiene tres rasgos principales: la libertad; la diferencia con la vida común (bajo la distinción entre tiempo de ocio y tiempo de trabajo) y el reconocimiento de límites tales como espacio y tiempo y a Caillois (1986), que agrega otras tres características: la incertidumbre de no conocer de antemano el resultado; la improductividad, al no pretender obtener lucro o bienes comerciales y sobre todo, la importancia de las reglas bajo las cuales funciona y organiza su dinámica; las cuales se mantienen vigentes a través del tiempo. (Enriz, 2014).

Interpretando a Secadas (1978) en su artículo "Las definiciones del juego" se puede entender que Piaget, considera que el juego es una actividad que tiene finalidad en sí mismo y en su desarrollo, a través de adjudicarse elementos y relaciones del entorno, buscando así generar condiciones placenteras para los participante al conjugar la ejecución, la simbología y la norma; también deriva que Vygotsky delimita al juego 
infantil como una actividad que no sólo es placentera sino que tiene objetivos socializantes ya que los juegos son siempre reglamentados y ocurren en un contexto sociocultural, siendo por ende integradores sociales y, el propio Secadas lo conceptúa como un accionar voluntario que se realiza en determinados espacios y tiempos, con reglas establecidas con anticipación, y fines primordialmente recreativos.

\section{El juego didáctico como medio para lograr una mayor autonomía}

Funcionalmente el juego puede ser espontaneo o contextual, cuando su objetivo primordial es su propio desarrollo como actividad recreativa y/o socializadora, o puede ser planificado o sistemático, cuando este se utiliza el aspecto recreativo como un medio o pretexto para lograr otros objetivos disímiles. En este sentido se puede definir como juego didáctico a toda actividad lúdica, sistematizada y ejecutada para alcanzar tanto metas de aprendizaje como crecimiento psicomotor $y / o$ cognitivo, teniendo orientaciones pedagógicas. Por tanto, puede afirmarse categóricamente que el juego didáctico es un importante medio o método de buscar que los pequeños infantes logren mejorar sus niveles de maduración al ofrecerles contenidos cognitivos, psicológicos, motores y socioafectivos.

Para Chacón (2008), el juego didáctico es una estrategia que se puede utilizar en cualquier nivel educativo porque posee un objetivo educativo, normas de ejecución e incluye momentos de acción pre reflexiva y de simbolización o apropiación abstractalógica de lo vivido para el logro de objetivos de enseñanza curriculares; con el fin de generar un aprendizaje efectivo a través de la diversión; esto es complementado por Bautista y López (2002) quienes catalogan como juego didáctico a aquel que combina aspectos lúdicos con los elementos de una organización eficiente de la enseñanza; tales como la participación, el dinamismo, el entrenamiento, la interpretación de papeles, la colectividad, la modelación, la retroalimentación, el carácter problémico, la obtención de resultados completos, la iniciativa, el carácter sistémico y la competencia.

Bajo la premisa de que todo sistema educativo tiene como finalidad primordial el formar un individuo integral, competente y feliz, es que el juego tiene un rol protagónico en el proceso educativo, ya sea por su carácter funcional y adaptativo y/o por ser una herramienta eficaz de aprendizaje y preparación para la vida de relación; en la medida que implica interactuar con el contexto y/o diversos actores, mediado por normas de desarrollo y convivencia que resultan placenteras a pesar de los factores externos; tal como afirma Gallardo \& Gallardo (2018) y Enriz (2014), quienes destacan que la actividad lúdica con fines educativos es un importante acotador conceptual si se utiliza con fines didácticos, afirmando que los individuos que juegan por obligación no están 
jugando al no tener libertad de decidir su participación, y que realmente están jugando son aquellos que lo hacen porque desean y encuentran placer en su participación, concluyendo que en esto consiste ser libre, es decir ser autónomo.

Mientras que Godoy (2015) llega a la conclusión que el juego didáctico produce beneficios motivacionales e instruccionales en los estudiantes ya que rompe con los esquemas tradicionales de enseñanza y permite crear su propio esquema de cómo resolver un ejercicio en específico y su relación con los aspectos teóricos; GarcíaEspinoza et al (2017), Solórzano (2017) y Ochoa (2018) coinciden que existe una influencia positiva del juego sobre el desarrollo de la autonomía; la que se desarrolla habilidades y prácticas por las cuales un niño vivencia y aprovecha sus experiencias, para tener más control y ser cada vez más independiente en sus relaciones, dominando progresivamente su cuerpo y de sus emociones, además de ser capaz de adaptar lo aprendido a nuevas situaciones; recalcando como componentes más significativos el sentido de iniciativa, el control de emociones y el aprendizaje significativo; lo que coincide con Olaya y Ramírez (2015), Carranza (2017) y Guamán y Venet (2019) quienes afirman que el juego como instancia de desarrollo de la autonomía facilitaría las condiciones para un aprendizaje significativo, es decir, situación en la cual los estudiantes transfieren conocimiento, de modo a aplicar a una situación inédita aquello que han aprendido en otros contextos.

Por tanto es necesario concentrar los esfuerzos en la utilización del juego como herramienta pedagógica indispensable en los niños, pues sirve para fortalecer la interacción social al generar espacios adecuados para el trabajo en equipo y, a partir de esta socialización en el marco de la cooperación, les permite adquirir y desarrollar actitudes y valores positivos; contribuyendo a mejorar el clima escolar al favorecer desarrollo cognitivo; siendo el juego el soporte pedagógico de los docentes para favorecer un aprendizaje significativo (Vera-Arcentales et al, 2021); esta premisa cobra mayor relevancia en el nivel preescolar, pues les permite tener mayor conciencia de sí mismo mientras va desarrollando su personalidad, habilidades psicomotoras y sensoriales (Nazario y Paredes, 2020), convirtiéndose en un mediador fundamental de las relaciones sociales con entre pares.

\section{Autonomía: libertad de decidir con responsabilidad y ser autosuficiente}

El autor, en una primera vertiente, considera que la autonomía, en los niños, es la capacidad de tomar decisiones enmarcadas dentro de la normativa socio-contextual, sin depender de la ayuda, vigilancia, control o de criterios ajenos, siendo un individuo capaz de asumir decisiones con libertad, responsabilidad y criterio propio. 
Por su parte Ternera (2010), asevera que los niños entre 3 y 7 años realizan significativos progresos en la capacidad de pensamiento, lenguaje y memoria, y una vez que aprenden a ejercer determinado grado de control consciente de sí y de su medio; mientras que, Sarramona (2011) afirma que la autonomía es una actitud o predisposición que una persona tiene hacia una determinada situación, la misma que lo conlleva a mantener un papel crítico de sus acciones referentes, en concordancia con un entorno social específico y con respeto a los demás;

Álvarez (2011) asevera que la autonomía es una aptitud que se va desarrollando desde los primeros años de vida en una relación conjunta con otros niños y adultos; aunque ambos autores coinciden que en esta etapa los infantes superan el egocentrismo de los primeros años de vida, lo que significa el respeta a valores como equidad, participación y justicia, reconociendo los derechos de otros; por lo que durante este proceso de adquisición de autonomía, el infante va siendo capaz de tomar sus propias decisiones y asumir la responsabilidad que esto implica, teniendo en cuenta las normas y reglas definidas para cada ocasión específica.

Mientras que, en una segunda vertiente, el autor afirma que autonomía en los niños, significa autosuficiencia funcional, lo que implica capacidad psicomotriz para realizar tareas diversas relacionadas al contexto temporal y espacial donde se desenvuelve; esto requiere reconocimiento de su esquema corporal, un determinado nivel de dominio físico y motor, adecuado a la acción a realizar y el necesario entendimiento del reto planteado por la tarea por cumplir.

Es por tanto indispensable buscar el mejoramiento de los ambientes de aprendizajes ya que en estos se suscitan situaciones que permiten que los niños vayan adquiriendo mayor conciencia de sus acciones y facilitan la incorporación de hábitos sociales, afectivos, comunicativos y de salud; permitiéndoles estructurarse como una persona autónoma, equilibrada, sana y capaz de sostener relaciones interpersonales significativas desde el marco del respeto; considerando en este proceso que el rol de docentes y padres es el de ser facilitadores y mediadores, entre los recursos y el ambiente de aprendizaje.

El juego didáctico, en este contexto, pretende mejorar la capacidad funcional de los pequeños estudiantes ya que esto les permitirá tener mayor autonomía; comparativamente es posible traer a colación el estudio de Campos y Mendoza (2020), quienes evidencian que el desarrollo de las habilidades funcionales favorece el desarrollo del nivel de autonomía en niños con síndrome de Down, destacando el papel 
de la familia y una intervención profesional temprana, para lograr se alcancen los objetivos de aprendizaje.

Por último León et al (2021) reafirman que en los niños pequeños, el proceso educativo está influenciado primordialmente por la teoría psicogenética de Piaget y la teoría socio-histórico-cultural de Vygotsky; por tanto este proceso formativo está estrechamente ligado con su contexto sociocultural, a partir del cual generan sus propios conocimientos, así como se integran a la sociedad y, es en este entorno que el juego se torna como un medio privilegiado para mejor su nivel de autonomía, mejorando su sentido de iniciativa, control emocional y aprendizaje significativo.

\section{MATERIALES Y MÉTODOS}

El desarrollo del presente estudio implicó la adopción de un modelo de revisión literaria crítica y reflexiva; este modelo pretende no sólo hacer una revisión de las publicaciones referentes al tema, sino también incluir reflexiones del autor sobre las mismas y concluir sobre su importancia, al mismo tiempo que se busca una expansión de la investigación. Esto favorece una rigurosidad científica basada en procedimientos heurísticos (Tamayo, 2004).

Por tanto, al ser una revisión reflexiva implica que el autor no sólo toma los conceptos de los artículos revisados, sino que pretende aportar opiniones y definiciones que considera relevantes; las mismas que figuran sin cita de autor. El proceso de revisión incluyó una etapa heurística de exploración rápida en bases de datos de Scopus, Ebsco, Scielo, Dialnet y Redalyc entre otros; también se complementó con una búsqueda en Google Académico, para mayor seguridad y afinar las referencias; en áreas relacionadas a ambas categorías: autonomía y juego didáctico.

Aunque en el proceso de recolección de información priorizó los artículos cuyo período o rango de publicaciones realizadas en la última década y que estuvieran directamente relacionadas con las mencionadas categorías, se consideraron algunos textos de mayor antigüedad, necesarios para aclarar y definir conceptos; se excluyeron todas las publicaciones que hacían referencia a juegos de competencia, juego profesional y, en autonomía, aquellos que no estaban vinculadas a los procesos educativos o de maduración infantil. Además de los términos centrales de búsqueda, se agregaron otros como "maduración" "juego y juego infantil", "tipos de juego", "importancia de la autonomía", "desarrollo de la autonomía"

Para los procesos de análisis de la información, se utilizaron matrices de integración para organizar los hallazgos y aportes de mayor relevancia y jerarquizarlos en 
concordancia con su mayor o menor relación a los temas centrales, para posteriormente analizarlos con mayor minuciosidad, buscando integrarlos. Se tomaron como ejes transversales de esta investigación; en base a la información procesada y al interés del autor, el juego, el juego didáctico, la autonomía y su interacción; para lo cual se elaboraron matrices de comparación de contenidos de los artículos escogidos y facilitar un entendimiento holístico de los temas.

\section{RESULTADOS}

El análisis e interpretación de la información obtenida se deriva de la conjugación de los más importantes hallazgos y aportes de los autores y se presentan como matrices organizadas de los mismos, permitiendo integrarlas con las opiniones del autor. De cada matriz se deriva un resultado como interpretación de la integración de conceptos vertidos en la tabla.

\section{Tabla 1}

Matriz de integración: Juego

Cuellar, Tenreyro Castellón

Enriz

Gallardo Gallardo.

Nazario y Paredes.

Piaget

Secadas

Vygotsky

Lip

Tiene carácter universal y puede ser considerado como un espacio, asociado a y la interioridad con situaciones imaginarias para suplir demandas culturales y para potenciar la lógica y la racionalidad.

Son ámbitos cuya principal finalidad es vincularse con normas y valores socialmente válidos.

Es vital e indispensable para el desarrollo de las personas, pues desarrolla el aspecto físico, emocional, cognitivo, afectivo, social y moral de niños; y favoreciendo su imaginación y creatividad, con la consecuente mejora de su capacidad para comunicarse y socializar; refuerza la atención y la memoria, despertando la curiosidad; mejora la autoestima, favoreciendo la aprehensión de valores, normas, roles, conductas, actitudes positivas; entre otros beneficios.

Permite a los niños, el tener mayor conciencia de sí mismo mientras van desarrollando su personalidad, habilidades psicomotoras y sensoriales, convirtiéndose en un mediador fundamental de las relaciones sociales con entre pares.

Actividad que tiene finalidad en sí mismo y en su desarrollo, a través de adjudicarse elementos y relaciones del entorno, buscando así generar condiciones placenteras para los participantes al conjugar la ejecución, la simbología y la norma;

Actividad voluntaria que se realiza en determinados espacios y tiempos, con reglas establecidas con anticipación y fines recreativos.

Actividad que además de ser placentera, tiene objetivos socializantes, al ser siempre reglamentados y ocurrir en un contexto sociocultural; siendo por ende integradores sociales.

Funcionalmente el juego puede ser espontaneo o contextual, cuando su objetivo primordial es su propio desarrollo como actividad recreativa y/o

ISSN: 1390-9320, Vol. 9, Núm. 1, febrero 2022 
socializadora, o puede ser planificado o sistemático, cuando este se utiliza el aspecto recreativo como un medio o pretexto para lograr otros objetivos disímiles.

Fuente: Elaboración Propia

El juego es una "actividad" psicomotora compleja, innata, voluntaria y divertida, que tiene como finalidad el juego en sí mismo, de participación voluntaria; es prioritariamente recreativo y socializante, pues se utilizan elementos y relaciones del contexto para lograr un acrecentamiento integral de los niños al favorecer mayor desarrollo físico, emocional, cognitivo, afectivo, social y moral; favoreciendo también su imaginación, creatividad y comunicación, su capacidad de atención y su memoria, conllevando al logro de una mayor autoestima al mejorar el conocimiento de sí mismo y de los demás.

El juego, a pesar de poder ser considerado como un espacio, asociado a la interioridad con situaciones imaginarias, tiende a potenciar la lógica y la racionalidad, ya que conjuga ejecución, simbología y normatividad; el mismo que al ocurrir en un contexto sociocultural, en determinado espacio - tempo y con reglas establecidas anticipadamente, favorece una mejor toma de conciencia en los niños con actitud positiva, valores y con respeto a sus pares y a las normas.

Funcionalmente el juego puede ser espontaneo o contextual, cuando su objetivo primordial es su propio desarrollo como actividad recreativa y/o socializadora, o puede ser planificado o sistemático, cuando este se utiliza el aspecto recreativo como un medio o pretexto para lograr otros objetivos disímiles.

\section{Tabla 2}

Matriz de integración: Juego didáctico

Principal estrategia en el proceso de enseñanza-aprendizaje en el nivel inicial y se constituye como una de las principales herramientas para favorecer la creatividad de los niños

$\begin{array}{ll}\text { Aboga por implementar los juegos didácticos, como metodología para la } \\ \text { Herrera. } & \text { enseñanza a nivel educativo; por ser parte importante en la vida de los }\end{array}$ niños, jóvenes y adultos.

Es aquel que combina aspectos lúdicos con los elementos de una

Bautista \& organización eficiente de la enseñanza; tales como la participación, el López. dinamismo, el entrenamiento, la interpretación de papeles, la colectividad, la modelación, la retroalimentación, el carácter problémico, la obtención de resultados completos, la iniciativa, el carácter sistémico y la competencia.

Chacón.

Estrategia que se puede utilizar en cualquier nivel educativo porque posee un objetivo educativo, normas de ejecución e incluye momentos de acción pre-reflexiva y de simbolización o apropiación abstracta-lógica de lo vivido 
para el logro de objetivos de enseñanza curriculares; con el fin de generar un aprendizaje efectivo a través de la diversión.

Produce beneficios motivacionales e instruccionales en los estudiantes ya

Godoy \& que rompe con los esquemas tradicionales de enseñanza y permite crear su Alberto. $\quad$ propio esquema de cómo resolver un ejercicio en específico y su relación con los aspectos teóricos.

Herramienta pedagógica indispensable en los niños, pues sirve para fortalecer la interacción social al generar espacios adecuados para el trabajo Vera- $\quad$ en equipo y, a partir de esta socialización en el marco de la cooperación, les Arcentales permite adquirir y desarrollar actitudes y valores positivos; contribuyendo a et al. mejorar el clima escolar al favorecer desarrollo cognitivo; siendo el juego el soporte pedagógico de los docentes para favorecer un aprendizaje significativo

Actividad lúdica, sistematizada y ejecutada para alcanzar tanto metas de aprendizaje como crecimiento psicomotor y/o cognitivo, teniendo orientaciones pedagógicas, que se constituye como un importante medio o método para lograr mejores niveles de maduración cuando se planifican adecuadamente sus contenidos cognitivos, psicológicos, motores y socioafectivos.

Fuente: Elaboración propia.

El juego didáctico es un tipo de actividad lúdica, sistematizada y ejecutada para alcanzar tanto metas de aprendizaje como crecimiento psicomotor y/o cognitivo, teniendo orientaciones pedagógicas y que combina aspectos lúdicos con los elementos de una organización eficiente de la enseñanza; tales como la participación, el dinamismo, el entrenamiento, la interpretación de papeles, la colectividad, la modelación, la retroalimentación, el carácter problémico, la obtención de resultados completos, la iniciativa, el carácter sistémico y la competencia; constituyéndose como un importante medio o método de buscar que los pequeños infantes logren mejorar sus niveles de maduración al ofrecerles contenidos cognitivos, psicológicos, motores y socio-afectivos, a su vez.

Como estrategia metodológica, se puede utilizar en cualquier nivel educativo porque posee un objetivo educacional, normas de ejecución e incluye momentos de acción prereflexiva y de simbolización o apropiación abstracta-lógica de lo vivido para el logro de objetivos de enseñanza curriculares; con el fin de generar un aprendizaje efectivo a través de la diversión y producir beneficios motivacionales e instruccionales, ya que rompe con los esquemas tradicionales de enseñanza permitiendo crear un esquema propio de cómo resolver un ejercicio en específico.

Es en sí, una herramienta pedagógica indispensable en los niños, pues sirve para fortalecer la interacción social al generar espacios adecuados para el trabajo en equipo y, a partir de esta socialización en el marco de la cooperación, les permite adquirir y 
desarrollar actitudes y valores positivos; contribuyendo a mejorar el clima escolar al favorecer desarrollo cognitivo; siendo el juego el soporte pedagógico de los docentes para favorecer un aprendizaje significativo

\title{
Tabla 3
}

Matriz de integración: Autonomía

\begin{abstract}
Los ambientes de aprendizajes suscitan situaciones que permiten que los niños Bedoya, vayan adquiriendo mayor conciencia de sus acciones y facilitan la incorporación Giraldo, de hábitos sociales, afectivos, comunicativos y de salud; permitiéndoles Montoya \& estructurarse como una persona autónoma, equilibrada, sana y capaz de Ramírez. sostener relaciones interpersonales significativas desde el marco del respeto; considerando en este proceso que el rol de docentes y padres es el de ser facilitadores y mediadores, entre los recursos y el ambiente de aprendizaje.

El desarrollo de las habilidades funcionales favorece el desarrollo del nivel de Campos \& autonomía en niños con síndrome de Down, destacando el papel de la familia Kam. y una intervención profesional temprana, para lograr se alcancen los objetivos de aprendizaje
\end{abstract}

Gómez-

Rijo, JiménezJiménez SánchezLa responsabilidad encargada en temas operativos y ambientales constituye López.

Reafirman que en los niños pequeños, el proceso educativo está influenciado primordialmente por la teoría psicogenética de Piaget y la teoría socio-históricocultural de Vygotsky; por tanto este proceso formativo está estrechamente León et al ligado con su contexto sociocultural, a partir del cual generan sus propios conocimientos, así como se integran a la sociedad y, es en este entorno que el juego se torna como un medio privilegiado para mejor su nivel de autonomía, mejorando su sentido de iniciativa, control emocional y aprendizaje significativo.

La base de sistemas éticos y filosóficos del mundo contemporáneo es el principio de autonomía y se define como la capacidad del hombre para tomar Mazo decisiones por sí mismo; por tanto, ser autónomo significa que el sujeto tiene capacidad y libertad para pensar por sí mismo, con sentido crítico y de acuerdo a un determinado contexto.

La importancia de la autonomía moral radica en que favorece la regulación de

Ochoa la propia conducta y la comprensión de las demás personas; permitiendo el establecimiento de una vida de relación pacífica y tolerante; por lo que se debe formar desde la primera infancia.

La autonomía se logra a través de un esfuerzo continuo y de refuerzo Sarramona permanente; siendo el objetivo principal de la educación, el logro de la autonomía personal

La autonomía es el derecho y la capacidad más importante en el desarrollo del ser humano, en cualquier contexto sociocultural, porque le permite autenticidad, crecimiento y libertad, en un clima de respeto a las personas y normas de convivencia. Puede ser definida como la capacidad de tomar decisiones enmarcadas dentro de la normativa socio-contextual, sin depender de la ayuda, 
vigilancia, control o de criterios ajenos, siendo un individuo capaz de asumir decisiones con libertad, responsabilidad y criterio propio.

Fuente: Elaboración propia.

La autonomía es el derecho y la capacidad más importante en el desarrollo del ser humano, en cualquier contexto sociocultural, porque le permite autenticidad, crecimiento y libertad, en un clima de respeto a las personas y normas de convivencia; por tanto se considera que el principio de autonomía es la base de los sistemas éticos y filosóficos del mundo contemporáneo y, puede ser definida como la capacidad del hombre para tomar decisiones por sí mismo, las mismas que deben estar enmarcadas con una normativa socio-contextual; por tanto ser autónomo significa que el sujeto tiene capacidad y libertad para pensar por sí mismo, con sentido crítico y, en concordancia con un determinado contexto; sin depender de la ayuda, vigilancia, control o criterios ajenos; significa también el tener la capacidad para asumir las consecuencias de sus decisiones con libertad, responsabilidad y criterio propio.

La importancia de la autonomía radica en que favorece la regulación de la propia conducta y la comprensión de las demás personas; permitiendo el establecimiento de una vida de relación pacífica y tolerante; por lo que se debe formar desde la primera infancia, a través de un esfuerzo continuo y del refuerzo permanente. Por otro lado, al estar el proceso formativo estrechamente ligado al contexto sociocultural, se generan conocimientos propios e integración social; siendo el objetivo principal de la educación, el logro de la autonomía personal.

Para el desarrollo del nivel de autonomía, especialmente en los infantes, se deben organizar los ambientes de aprendizajes para suscitar situaciones que les permitan adquirir progresivamente una mayor conciencia de sus acciones y les faciliten la incorporación de hábitos sociales, afectivos, comunicativos y de salud; permitiéndoles, a su vez, estructurarse como una persona autónoma, equilibrada, sana y capaz de sostener relaciones interpersonales significativas desde el marco del respeto. Se debe considerar que en este proceso que, el rol de docentes y padres es el de ser facilitadores y mediadores, entre los recursos y el ambiente de aprendizaje.

Otros medios que favorecen este crecimiento son el desarrollo de las habilidades funcionales, el delegar responsabilidad en temas operativos y ambientales y, la utilización del juego como medio didáctico. 


\section{DISCUSIÓN}

Las nociones de juego y autonomía son variadas y múltiples, por ser términos polisémicos, de gran alcance o amplitud. En primera instancia, para definir juego no sólo se considera las ideas vertidas en este estudio, pues en todos los sistemas socioculturales de la actualidad, el juego no tiene que ver sólo con los niños, sino con todas y cada una de las personas; siendo relacionada intrínsecamente a la idea del deporte y la competición, por tanto tampoco tiene solamente finalidad recreativa, al ser una actividad profesional que requiere y genera grandes inversiones de dinero, constituyéndose como una actividad generadora de trabajo, planificada y organizada.

En este sentido el presente estudio se delimita al juego infantil, de carácter recreativo y no deportivo; teniendo en el juego didáctico su centro de atención; diferenciándolos (juego y juego didáctico) en cuanto a su funcionalidad, ya que el segundo, a pesar de tener todas las características del primero, se utiliza metodológicamente con fines educativos; es decir para que los infantes alcancen logros de aprendizaje y se reflejen en cambios positivos de conducta, respecto al ser autónomos. En cuanto al juego y juego didáctico, el autor observa que los investigadores analizados son congruentes entre sí en cuanto a su definición, funciones y características y, concuerda plenamente con ellos, lo que se evidencia en las conclusiones a las que arriba con respecto a estos temas.

En cuanto a lo referente a la autonomía, se puedo encontrar que está abarca desde las personas hasta las instituciones y países, que es un principio ético y filosófico (Álvarez, 2011), un fin primordial de la educación (Kamii, 2000) y que puede ser tipificada o clasificada en concordancia con un contexto específico. Por tanto, también se delimitó el estudio al análisis de la autonomía en niños, de su significado, el cómo incrementarla y su importancia. Se encontró coincidencia tanto en las definiciones del término, como en sus alcances y en que la utilización del juego didáctico es un valioso instrumento pedagógico que beneficia la adquisición de autonomía cognitiva, psicosocial y funcional.

\section{CONCLUSIONES}

El juego es una "actividad" psicomotora compleja, innata, voluntaria y divertida, que tiene como finalidad el juego en sí mismo, de participación voluntaria; es primordialmente recreativo y socializante, que favorecer el desarrollo físico, emocional, cognitivo, afectivo, social y moral; así como su imaginación, creatividad, comunicación, capacidad de atención y memoria; logrando en los niños conllevando una mayor autoestima y mejor conocimiento de sí mismo y de los demás. El juego, favorece la 
sociabilización al ocurrir en un contexto sociocultural y potencia la lógica y la racionalidad al conjugar ejecución, simbología y normatividad;

El juego didáctico es un tipo de actividad lúdica, sistematizada y ejecutada para alcanzar tanto metas de aprendizaje como crecimiento psicomotor y/o cognitivo, teniendo orientaciones pedagógicas y que combina aspectos lúdicos con los elementos de una organización eficiente de la enseñanza; se emplea como un importante medio o método para que los pequeños infantes aceleren sus procesos de maduración al ofrecerles contenidos cognitivos, psicológicos, motores y socioafectivos. Es también una estrategia metodológica utilizable en todo nivel educativo ya que tiene componentes de acción pre reflexiva y de simbolización o apropiación abstracta-lógica de lo vivido para el logro de objetivos de enseñanza curriculares. Es una herramienta pedagógica que sirve para fortalecer la interacción social al generar espacios adecuados para el trabajo en equipo, la cooperación mutua y aprendizajes significativos entre otros.

La autonomía es el derecho innato de toda persona a ser libre, debiendo ser ejercido y respetado en cualquier contexto sociocultural, siendo sinónimo de autenticidad, crecimiento y libertad, enmarcado en un clima de respeto a las personas y normas de convivencia; puede ser definida como la capacidad para tomar decisiones por uno mismo, en concordancia con una normativa socio-contextual; por tanto ser autónomo significa que el sujeto tiene capacidad y libertad para pensar por sí mismo, con sentido crítico, sin depender de la ayuda, vigilancia, control o criterios ajenos; significa también el tener la capacidad para asumir las consecuencias de sus decisiones con libertad, responsabilidad y criterio propio. Esta autonomía debe ser trabajada desde la primera infancia en forma sistemática, organizando ambientes de aprendizajes que susciten situaciones problématizadoras y les faciliten la incorporación de valores sociales, afectivos, comunicativos y de salud, en conjunción son padres de familia y docentes.

Existe una fuerte relación entre el juego y el desarrollo del nivel de autonomía en los niños; por ende, los logros de aprendizaje en lo referente a la autonomía deben ser encausados mediante juegos motores, de atención, razonamiento e imaginación. El juego didáctico es la mejor herramienta pedagógica para alcanzar este fin; por tanto, es recomendable que, en la formación de docentes especializados en educación inicial, se le tenga como un eje transversal y en constante desarrollo, reforzando y actualizando continuamente sus competencias didácticas.

Es recomendable tener en cuenta la importancia de desarrollar la autonomía lo más temprano posible, tanto en el hogar como en nidos, guarderías e instituciones educativas de nivel inicial y primaria, para lo cual es indispensable un cambio de 
mentalidad tanto en padres como en maestros; para quienes la autonomía debe desarrollarse en niveles educativos avanzados y concientizarlos en la utilización de los juegos, no sólo como una actividad recreativa, sino también de gran utilidad formativa y educativa.

\section{REFERENCIAS BIBLIOGRÁFICAS}

Albornoz Zamora, E. J. (2019). El juego y el desarrollo de la creatividad de los niños/as del nivel inicial de la escuela Benjamín Carrión. Conrado, 15(66), 209-213.

Álvarez, H. M. M. (2012). La autonomía: principio ético contemporáneo. Revista Colombiana de Ciencias Sociales, 3(1), 115-132.

Bautista Vallejo, J. M., \& López, N. R. (2002). El juego didáctico como estrategia de atención a la diversidad.

Bedoya, L., Giraldo, A., Montoya, N., \& Ramírez, L. M. (2013). La autonomía en la primera infancia desde el trabajo por proyectos.

Caillois, R. (1986). Los juegos. México: FCE. Recuperado el, 27.

Campos García, K. V., \& Kam Mendoza, S. E. (2020). Desarrollo de Habilidades Funcionales para la autonomía en niños de 8 años con Síndrome de Down de Escuela Hogar Nazareth en Guayaquil (Bachelor's thesis, Universidad de Guayaquil-Facultad de Ciencias Psicológicas)

Carranza Alcántar, M. D. R. (2017). Significant teaching and learning in a blended learning: perceptions of teachers and students. RIDE. Revista Iberoamericana para la Investigación y el Desarrollo Educativo, 8(15), 898-922.

Chacón, P. (2008). El Juego Didáctico como estrategia de enseñanza y aprendizaje. ¿Cómo crearlo en el aula? Nueva aula abierta, 16(5), 1-8.

Cuellar Cartaya, M. E., Tenreyro Mauriz, M., \& Castellon Leon, G. (2018). The game in preschool education. Historical foundations. Revista conrado, 14(62), 117-123.

Enriz, N. (2014). Juego, concepto y ordenamiento de una práctica escurridiza. Espacios en Blanco. Revista de Educación, (24), 17-33.

Gallardo-López, J. A., \& Gallardo Vázquez, P. (2018). Teorías sobre el juego y su importancia como recurso educativo para el desarrollo integral infantil. Revista Educativa Hekademos, 24(11), 41-51. 
García Espinoza, M., Ortiz Cárdenas, T., \& Chávez Loor, M. D. (2017). Estrategias orientadas al aprendizaje autónomo en la Universidad Estatal Península de Santa Elena, Ecuador. Revista Cubana de Educación Superior, 36(3), 74-84.

Godoy, M., \& Alberto, K. (2015). Aplicación de un juego didáctico como estrategia pedagógica para la enseñanza de la estequiometría. Revista de investigación, 39(84), 181-204.

Gómez-Rijo, A., Jiménez-Jiménez, F. y Sánchez-López, C. (2015). Development of Autonomy of Elementary Students in Physical Education through a process of action research. Revista Internacional de Ciencias del Deporte, 11 (42), 310-328. http://dx.doi.org/10.5232/ricyde2015.04201

Guamán Gómez, V. J., \& Venet Muñoz, R. (2019). El aprendizaje significativo desde el contexto de la planificación didáctica. Conrado, 15(69), 218-223.

Herrera, B. M. (2017). Aplicación de juegos didácticos como metodología de enseñanza: Una Revisión de la Literatura. Pensamiento Matemático, 7(1), 75-92.

Huizinga, J. (2014). Homo ludens ils 86. Routledge.

Kamii, C. (2000). La autonomía como finalidad de la Educación: implicaciones de la Teoría de Piaget. Secretaría de Educación y Cultura-Dirección de Currículo. Universidad de Illinois, Círculo de Chicago.

León Roldán, Z. E., Méndez Vergaray, J., Campos Valdivia, S. T., Arteaga, N., \& Elizabeth, M. (2021). Las actividades lúdicas en la adquisición de la autonomía de niños de preescolar.

Ministerio de Educación del Perú. MINEDU. (2017). Fundamentos de la educación inicial Introducción. https://ugelsurcubamba.com/ministerio/wpcontent/uploads/Fundamentos-de-la-educación-inicial.pdf.

Nazario, M.; Paredes, M. (2020). El juego en la identidad y autonomía del niño. UCV HACER. Revista de Investigación y Cultura, 9 (1), 11-17. En https://n9.cl/iyyzm.

Ochoa Montiel, F. J. (2018). La formación de la autonomía moral desde el preescolar. Varona. Revista Científico Metodológica, (66).

Olaya, A., \& Ramírez, J. (2015). On the Traces of significant learning, alternative and innovation in knowledge and pedagogical practice. Revista Científica Guillermo de Ockham, 13(2), 117-125.

Ruiz Gutiérrez, M. (2017). El juego: Una herramienta importante para el desarrollo integral del niño en Educación Infantil. 
Sarramona, J. (2011). Autonomía y calidad de la educación. [Conferencia dictada en el XII Congreso Internacional de Teoria de la Educación]. Universidad de Barcelona. http://www.cite2011.com/Ponencias/JSarramona.pdf

Secadas Marcos, F. (1978). Las definiciones del juego. En https://n9.cl/y46q0.

Solórzano, Y. (2017). Autonomous learning and skills. Dominio de las Ciencias, 3, 241253. https://dialnet.unirioja.es/servlet/articulo?codigo $=5907382$

Tamayo y Tamayo, M. (2004). El proceso de la investigación científica. Editorial Limusa.

Ternera, L. A. C. (2010). Relación entre el desarrollo personal social y los procesos evolutivos vinculados con el aprendizaje escolar en las áreas del lenguaje y la cognición. Psicogente, 13(23).

Vera-Arcentales, F. O., del Carmen Párraga-Salvatierra, N., Bazurto-Briones, N. A., Mendoza-Castro, A. M., \& Barcia-Briones, M. F. (2021). El juego como estrategia psicopedagógica y su impacto en la calidad educativa de los estudiantes de básica media. Dominio de las Ciencias, 7(1), 903-919. 